\title{
Evolution-Free Hamiltonian Parameter Estimation through Zeeman Markers
}

\author{
Daniel Burgarth ${ }^{*}$ \\ Institute of Mathematics, Physics and Computer Science, Aberystwyth University, \\ Aberystwyth SY23 3BZ, United Kingdom \\ Ashok Ajoy \\ Department of Chemistry, University of California Berkeley, and Materials Science Division, \\ Lawrence Berkeley National Laboratory, Berkeley, California 94720, USA
}

(Received 31 December 2016; published 21 July 2017)

\begin{abstract}
We provide a protocol for Hamiltonian parameter estimation which relies only on the Zeeman effect. No time-dependent quantities need to be measured; it fully suffices to observe spectral shifts induced by fields applied to local "markers." We demonstrate the idea with a simple tight-binding Hamiltonian and numerically show stability with respect to Gaussian noise on the spectral measurements. Then we generalize the result to show applicability to a wide range of systems, including quantum spin chains, networks of qubits, and coupled harmonic oscillators, and suggest potential experimental implementations.
\end{abstract}

DOI: 10.1103/PhysRevLett.119.030402

Introduction.-One of the most fundamental concepts of quantum theory is the Hamiltonian as the generator of dynamics. Hamiltonians are of paramount importance in our understanding of matter and its properties, but they can also do some work for us: in quantum technology, they can trigger quantum simulations or even form the basis of quantum computing. The current drive towards highfidelity quantum devices has kindled renewed interest in Hamiltonian parameter estimation [1].

Hamiltonian parameter estimation is important but costly in terms of resources used. It requires detailed control and measurements, as well as extensive postprocessing of the measured data. In specific situations, however, specialized protocols can ease the task. As such, indirect estimation has recently been developed [2] to allow parameter estimation in systems with limited access. The basic idea is to measure a free induction decay of the type $f(t)=\langle 1|\exp (-i H t)| 1\rangle=$ $\sum_{k} \exp \left(-i e_{k} t\right)\left|\left\langle 1 \mid e_{k}\right\rangle\right|^{2}$ with respect to some reference state $|1\rangle$ representing a "local" probe. Fourier transform then provides the spectrum $\left\{e_{k}\right\}$ and the coefficients $\left|\left\langle 1 \mid e_{k}\right\rangle\right|$, and it was shown that for one-dimensional systems these limited data can suffice to estimate the full system.

This initial work has been extended in several directions. It was shown [3], perhaps surprisingly, that for certain systems the initialization in a reference state is not necessary. If more than one probe can be used, the method can be applied to arbitrary networks [4]. Other physical systems, such as fermionic and bosonic networks [5] and linear passive

Published by the American Physical Society under the terms of the Creative Commons Attribution 4.0 International license. Further distribution of this work must maintain attribution to the author(s) and the published article's title, journal citation, and DOI. systems [6], were also found to be indirectly estimable. It was found that additional control of the probes helps to gain phase information [7], and a description in terms of polynomial equations was developed in Refs. [8-10]. Graph structures occurring in biometric systems were studied in Ref. [11], and the first NMR experiments demonstrated feasibility of the method for small systems $[12,13]$.

All of these methods suffer from a major drawback: the requirement to measure time-resolved dynamics and to do so locally. This is hard, because the dynamics can be very fast for strongly coupled systems, because decoherence limits the time period over which useful data can be acquired, and because local measurements are difficult. It is interesting to note, however, that half of the required data for the above schemes, namely, the spectrum $\left\{e_{k}\right\}$, can be relatively easy to measure. The spectrum is a global property of a many-body system and can be measured by absorption or emission of electromagnetic radiation, and many advanced methods for spectroscopy in a plethora of experiments have been established. Generally, spectroscopy does not require time-resolved measurements and can work well in the presence of decoherence. Does the spectrum provide enough information to perform Hamiltonian parameter estimation? As can be seen from Ref. [2], one fixed spectrum can give rise to infinitely many different parameter choices (through fixing $\left\langle 1 \mid e_{k}\right\rangle$ ). However, we show in this Letter that two spectra do the job: one being the original spectrum of $H$, and one being the spectrum of a modified Hamiltonian $H^{\prime}$ which arises from applying a local field to a probe that we call the "Zeeman marker." This probe does not have to be measured locally and no time-dependent data are required. We first demonstrate this idea with a simple (but common) tightbinding Hamiltonian, then analyze its stability, and finally generalize to spin Hamiltonians, free fermions and bosons, 
and arbitrary networks. Finally we suggest potential experimental implementations.

Simple model.-We consider an $N$-dimensional Hilbert space with basis $\{|1\rangle,|2\rangle, \ldots,|N\rangle\}$ and a tight-binding Hamiltonian given by

$$
H=\sum_{n=1}^{N-1} c_{n}|n\rangle\left\langle n+1\left|+c_{n}^{*}\right| n+1\right\rangle\langle n| .
$$

Although in general the parameters $c_{n}$ could be complex, it suffices to consider the case $c_{n}>0$, because complex phases $e^{i \phi_{n}}$ can always be absorbed in the choice of basis, $\left\{|2\rangle \rightarrow e^{-i \phi_{1}}|2\rangle,|3\rangle \rightarrow e^{-i\left(\phi_{2}+\phi_{1}\right)}|3\rangle, \ldots,|N\rangle \rightarrow e^{-i \sum_{n=1}^{N-1} \phi_{n}}\right\}$. Our target is to estimate the parameters $c_{n}$ by measuring the spectrum of the system only. In order to do so, let us apply a field $f|1\rangle\langle 1|$ at site 1 (see Fig. 1). Thus, we have modified our Hamiltonian to $H^{\prime}=H+f|1\rangle\langle 1|$. Denoting the spectrum of the original Hamiltonian $H$ as $\left\{e_{k}\right\}$ and the one of $H^{\prime}$ as $\left\{e_{n}^{\prime}\right\}$, we can derive [14]

$$
\left|\left\langle e_{k} \mid 1\right\rangle\right|^{2}=\left(e_{k}^{\prime}-e_{k}\right) / f \prod_{m \neq k} \frac{\left(e_{k}-e_{m}^{\prime}\right)}{\left(e_{k}-e_{m}\right)} .
$$

Without loss of generality we may choose the phases of the eigenstates $\left|e_{k}\right\rangle$ such that $\left\langle e_{k} \mid 1\right\rangle \geq 0$. This means that the measurements of the spectra of $H$ and $H^{\prime}$ reveal $\left\langle e_{k} \mid 1\right\rangle$. We remark that the value of $f$ plays no significant role and indeed can be unknown: the $\left|\left\langle e_{k} \mid 1\right\rangle\right|^{2}$ sum up to 1 , which implies that $f$ can also be inferred from the spectra.

From $\left\langle e_{k} \mid 1\right\rangle$ we can obtain the $c_{n}$ following Refs. $[2,8]$ : the equation

$$
\sum_{k=1}^{N} e_{k}^{m}\left|\left\langle 1 \mid e_{k}\right\rangle\right|^{2}=\left\langle 1\left|H^{m}\right| 1\right\rangle
$$

provides iterative polynomial equations in the $c_{n}$ which can be solved; e.g., for $m=2$, we get $c_{1}^{2}$, for $m=4$, $c_{1}^{2}\left(c_{1}^{2}+c_{2}^{2}\right)$, and so on. We remark that on-site terms of the form $b_{n}|n\rangle\langle n|$ can also be estimated [2]. A more thorough analysis of such polynomials in terms of their Gröbner basis was recently provided in Ref. [10].

Stability.-We numerically analyze the stability of the algorithm with respect to errors in the spectroscopy.

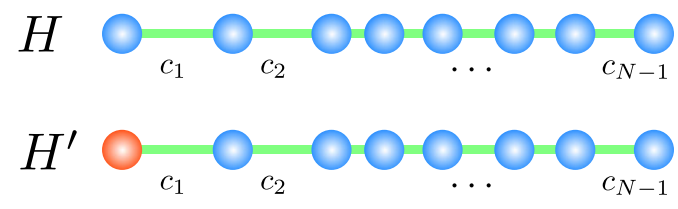

FIG. 1. We estimate the parameters $c_{n}$ of a Hamiltonian $H$ given by Eq. (1) by actively modifying it to $H^{\prime}$, applying a timeindependent local field on the "Zeeman marker" (orange). Performing spectroscopy on $H$ and $H^{\prime}$ yields two spectra, $\left\{e_{k}\right\}$ and $\left\{e_{n}^{\prime}\right\}$, from which we compute the $c_{n}$ through Eqs. (2) and (3).
Modeling the error as independent Gaussian noise, we find that the error scales weakly with the chain length up to a critical value, after which the estimation scheme fails (see Fig. 2). For the chains considered, the spectrum is bounded by the interval $[-2,2]$ and the maximum eigenvalue is close to 2. Thus, a standard deviation in the Gaussian noise of 0.04 corresponds to a percentage of the range of eigenvalues measured. As we can see, the errors must be below that order of magnitude to give useful estimation results for chains of length range up to $N=20$. As a rough argument, the error must be smaller than the smallest difference in eigenenergies, which scales as $2 / N^{2}$ for isotropic chains. The corresponding critical values $N_{c}=5,7,10,14$ roughly match the numerical observations. Although in the general scheme the value of the parameter $f$ is not important, for stability it is clear that we want $f$ as large as possible to get a big spectral difference between $H$ and $H^{\prime}$. This is confirmed by numerics, which scales best when $f \gg 1$ (we found the error saturates after approximately $a=10$ ).

Generalizations. - The simple model discussed above can easily be extended to other interesting cases. First, consider Heisenberg spin Hamiltonians of the form

$$
H=\sum_{n=1}^{N} c_{n}(X X+Y Y+\Delta Z Z)_{n, n+1}+\sum_{n=1}^{N} b_{n} Z_{n} .
$$

These models conserve the total number of excitations. Considering the sector with one excitation, they become equivalent to the tight-binding models discussed above. If one can either initialize such a system in the first excitation sector or select the spectral lines corresponding to the first excitation sector using the usual Zeeman effect, then the above protocol becomes applicable.

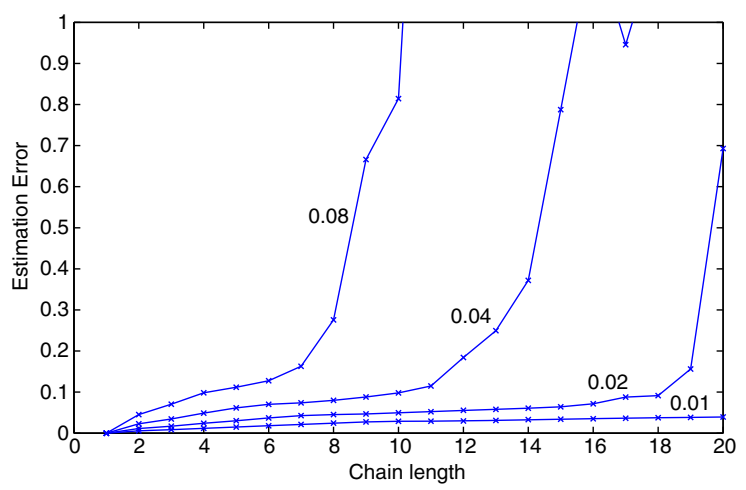

FIG. 2. Average error in the estimation of the $c_{n}$ in Eq. (1). Shown is $\sum_{n=1}^{N-1}\left(c_{n}-\hat{c}_{n}\right)^{2} /(N-1)$ averaged over 10000 samples for chains of length $N=2, \ldots, 20$. The true couplings were taken to be $c_{n}=1$ and the estimated couplings $\hat{c}_{n}$ were numerically computed using the tomography scheme described in the text. The field parameter was chosen to be large $(f=10)$. The four lines correspond to Gaussian noise in the spectral measurements of standard deviations $0.01-0.08$. 
Likewise, for quadratic Hamiltonians of the form

$H=\sum_{n=1}^{N-1} A_{n, n+1} a_{n}^{\dagger} a_{n+1}+\frac{1}{2} \sum_{n=1}^{N-1}\left(B_{n, n+1} a_{n}^{\dagger} a_{m+1}^{\dagger}+\right.$ H.c. $)$,

with $A$ Hermitian and $B^{T}=-\epsilon B$ for fermions $(\epsilon=1)$ and bosons $(\epsilon=-1)$, we can follow a similar protocol to Ref. [5] by applying rank-one perturbations $f a_{1}^{\dagger} a_{1}$ and $g a_{1}^{\dagger} a_{1}^{\dagger}+$ H.c. followed by spectroscopy.

The final generalization is to arbitrary networks. Because the derivation of Eq. (2) is not specific to the choice of $|1\rangle\langle 1|$ as a field, we can consider using different perturbations. In particular, applying $|\psi\rangle\langle\psi|$ followed by spectroscopy provides us with $\left|\left\langle e_{k} \mid \psi\right\rangle\right|^{2}$. We choose four different operations $|\psi\rangle=|n\rangle,|m\rangle,(|n\rangle+|m\rangle) / \sqrt{2},(|n\rangle+i|m\rangle) / \sqrt{2}$ followed by spectroscopy. This provides us with $\left|\left\langle n \mid e_{k}\right\rangle\right|^{2},\left|\left\langle m \mid e_{k}\right\rangle\right|^{2}$, $\left|\left\langle n \mid e_{k}\right\rangle\right|^{2}+\left|\left\langle m \mid e_{k}\right\rangle\right|^{2}+\left\langle n \mid e_{k}\right\rangle\left\langle e_{k} \mid m\right\rangle+\left\langle m \mid e_{k}\right\rangle\left\langle e_{k} \mid n\right\rangle$, and $\left|\left\langle n \mid e_{k}\right\rangle\right|^{2}+\left|\left\langle m \mid e_{k}\right\rangle\right|^{2}-i\left\langle n \mid e_{k}\right\rangle\left\langle e_{k} \mid m\right\rangle+i\left\langle m \mid e_{k}\right\rangle\left\langle e_{k} \mid n\right\rangle$, from which $\left\langle e_{k} \mid n\right\rangle$ and $\left\langle e_{j} \mid m\right\rangle$ can be estimated. Applying these fields on all pairs $(n, m)$ of the graph would therefore provide all eigenstates and thus the full Hamiltonian.

But we can do much better: we only need to apply the fields on a subset of the full system, provided that they form an "infecting set" [4] (also known as a "zero-forcing set" in graph theory [18]) and provided that the couplings are positive. Roughly speaking, it suffices to apply fields to the "surface" of the graph. Under those conditions, the protocol provided in Ref. [4] combined with the above can be applied to estimate all parameters. We can think of such a set as "marker" particles which respond to external perturbations with a Zeeman shift to provide the parameters of the Hamiltonian.

Experimental implementations.-We now consider experimental systems where our Hamiltonian estimation protocol with Zeeman markers can be realized, along with potential applications.

Chains of ions trapped in rf-Paul traps [19] are a versatile platform for quantum simulation [20]. Indeed, one can engineer Mølmer-Sørensen Hamiltonians [21] of the type $H=\sum_{i j} J_{i j}\left(S_{i}^{+} S_{j}^{-}+\right.$H.c. $)$, and retain only nearest-neighbor interactions via Floquet Hamiltonian engineering [22] techniques, for instance, using laser Stark-shift gradients. Building a versatile quantum simulator relies on a precise characterization of the Hamiltonian between the ions of the chain, which to a good approximation is $J_{i j} \propto \sum_{k}\left[\left(b_{i}^{k} b_{j}^{k}\right) /\left(\mu^{2}-\omega_{k}^{2}\right)\right]$ - a function of coupling $b^{k}$ to the collective phonon eigenmodes $\omega_{k}$ that mediate the spin interactions, and where $\mu$ is the laser detuning [23]. Measuring the couplings accurately will allow one to experimentally verify and benchmark this calculated expression and identify correction terms given by residual phonon contributions [24]. Overall, such Hamiltonian identification will enable the directed engineering of exotic Hamiltonians and ground states in an ion trap system [25]. The protocol with Zeeman markers is especially suited since one could prepare and manipulate end states of the chain optically and perform spectroscopy [26].

The protocol might also find important application in characterizing spin-based quantum chains, consisting of either nuclear or electron spins. Such chains have been proposed as test beds for quantum simulation and quantum transport, and as "wires" to link distributed quantum registers [27]. Nuclear chains occur in a variety of natural systems, for example, ${ }^{19} \mathrm{~F}$ chains in solid crystals of Fluorapatite [28] and ${ }^{13} \mathrm{C}$ spins in certain alkane backbones [29]. In the latter, the spins are coupled by electron-mediated $J$ couplings, which at zero or ultralow fields intrinsically has the Heisenberg form $H=\sum_{j}\left(J_{j} \vec{I}_{j} \cdot \vec{I}_{j+1}+B I_{z j}\right)$, where one assumes a weak field $B \ll J_{j}$ is applied [30]. Zeeman markers can be readily applied if the ends of the chain are a different nuclear species. Spectroscopy of the chain eigenmodes can be achieved by preparing initial states that have support on all the eigenstates-a simple example being a polarized spin at particular location in an otherwise mixed spin chain - and subsequent readout. Selective preparation of such states can, for instance, be done via algorithmic cooling [31] or targeted hyerpolarization techniques $[32,33]$. Spectroscopic readout can be performed directly at zero field [34] or by field cycling to higher fields [35,36], each revealing spin transitions from which the Hamiltonian eigenvalues can be reconstructed.

In addition, we envision complementary applications for electron spin chains constructed out of nitrogen-vacancy (NV) centers and P1 centers in diamond. Controlled nitrogen-ion implantation allows the deterministic creation of such spin chains, with spacings under $40 \mathrm{~nm}$ [37], and finite conversion efficiencies determine the ratio of $\mathrm{NV}$ and $\mathrm{P} 1$ sites. The NV centers can be optically polarized and read out, while $\mathrm{P} 1$ centers are not directly addressable at the single spin level. Such chains have found wide interest in quantum information [38,39] and in environment assisted quantum sensing [40], where the P1 centers can act to amplify the magnetic field sensitivity of the NV centers [41]. The sensitivity gains could be significant-approaching close to the Heisenberg limit in some protocols [40]—and allowing a plethora of applications in nanoscale magnetometry $[42,43]$. However, in practice, the poor characterization of the couplings between "dark" P1 spins has been the major obstacle - a problem that would be exactly addressed by our method. Moreover, since the protocol reveals both the couplings as well as on-site fields, it could enable arrayed quantum sensing using an electron spin chain.

Conclusions. - The Hamiltonian parameter estimation demonstrated above relies on the ability to actively modify the system through the application of local fields. These do not need to be time dependent, nor do the measurements need to resolve the dynamics. This paves the way to a stable and general parameter estimation. In some systems one might even get away without having to apply local fields, 
by instead engineering two different Hamiltonians $H$ and $H^{\prime}$, which differ only locally, e.g., by attaching a chemical ligand to a certain atom or by adding or removing particles.

An important result from quantum computing [44] shows that, in principle, very hard ("QMA-complete") problems can be encoded in spectral properties of simple Hamiltonians. From a broader perspective, it is fascinating to speculate which other dynamical properties can be mapped into spectral ones.

D. B. acknowledges support from the EPSRC Grant No. EP/M01634X/1 and from BQIC. We thank R. Islam and K. B. Whaley for stimulating discussions.

*Corresponding author.

daniel@burgarth.de

[1] J.H. Cole, Hamiltonian tomography: the quantum (system) measurement problem, New J. Phys. 17, 101001 (2015).

[2] D. Burgarth, K. Maruyama, and F. Nori, Coupling strength estimation for spin chains despite restricted access, Phys. Rev. A 79, 020305 (2009).

[3] C. Di Franco, M. Paternostro, and M. S. Kim, Hamiltonian Tomography in an Access-Limited Setting without State Initialization, Phys. Rev. Lett. 102, 187203 (2009).

[4] D. Burgarth and K. Maruyama, Indirect Hamiltonian identification through a small gateway, New J. Phys. 11, 103019 (2009).

[5] D. Burgarth, K. Maruyama, and F. Nori, Indirect quantum tomography of quadratic Hamiltonians, New J. Phys. 13, 013019 (2011).

[6] M. Guta and N. Yamamoto, System identification for passive linear quantum systems, IEEE Trans. Autom. Control 61, 921 (2016).

[7] D. Burgarth and K. Yuasa, Quantum System Identification, Phys. Rev. Lett. 108, 080502 (2012).

[8] J. Zhang and M. Sarovar, Quantum Hamiltonian Identification from Measurement Time Traces, Phys. Rev. Lett. 113, 080401 (2014).

[9] J. Zhang and M. Sarovar, Identification of open quantum systems from observable time traces, Phys. Rev. A 91, 052121 (2015).

[10] A. Sone and P. Cappellaro, Hamiltonian identifiability assisted by a single-probe measurement, Phys. Rev. A 95, 022335 (2017).

[11] K. Maruyama, D. Burgarth, A. Ishizaki, K. B. Whaley, and T. Takui, Application of indirect Hamiltonian tomography to complex systems with short coherence times, Quantum Inf. Comput. 12, 763 (2012).

[12] S.-y. Hou, H. Li, and G.-L. Long, Experimental quantum Hamiltonian identification from measurement time traces, Science Bulletin 62, 863 (2017).

[13] E. H. Lapasar, K. Maruyama, D. Burgarth, T. Takui, Y. Kondo, and M. Nakahara, Estimation of coupling constants of a three-spin chain: a case study of Hamiltonian tomography with nuclear magnetic resonance, New J. Phys. 14, 013043 (2012).
[14] See Supplemental Material at http://link.aps.org/ supplemental/10.1103/PhysRevLett.119.030402 for a proof, which includes Refs. [15-17].

[15] E. N. Economou, Green's Functions in Quantum Physics (Springer, Berlin, Heidelberg, 2005).

[16] G. M. L. Gladwell, Inverse Problems in Vibration (Springer Netherlands, Dordrecht, 2005).

[17] V. Giovannetti and D. Burgarth, Improved Transfer of Quantum Information Using a Local Memory, Phys. Rev. Lett. 96, 030501 (2006).

[18] F. Barioli, W. Barrett, S. M. Fallat, H. T. Hall, L. Hogben, B. Shader, P. van den Driessche, and H. van der Holst, Zero forcing parameters and minimum rank problems, Linear Algebra Appl. 433, 401 (2010).

[19] C. Monroe, Quantum information processing with atoms and photons, Nature (London) 416, 238 (2002).

[20] K. Kim, M.-S. Chang, S. Korenblit, R. Islam, E. Edwards, J. Freericks, G.-D. Lin, L.-M. Duan, and C. Monroe, Quantum simulation of frustrated Ising spins with trapped ions, Nature (London) 465, 590 (2010).

[21] A. Sørensen and K. Mølmer, Quantum Computation with Ions in Thermal Motion, Phys. Rev. Lett. 82, 1971 (1999).

[22] A. Ajoy and P. Cappellaro, Quantum Simulation via Filtered Hamiltonian Engineering: Application to Perfect Quantum Transport in Spin Networks, Phys. Rev. Lett. 110, 220503 (2013).

[23] D. Porras and J. I. Cirac, Effective Quantum Spin Systems with Trapped Ions, Phys. Rev. Lett. 92, 207901 (2004).

[24] C.-C. Joseph Wang and J. K. Freericks, Intrinsic phonon effects on analog quantum simulators with ultracold trapped ions, Phys. Rev. A 86, 032329 (2012).

[25] R. Islam, C. Senko, W. Campbell, S. Korenblit, J. Smith, A. Lee, E. Edwards, C.-C. Wang, J. Freericks, and C. Monroe, Emergence and frustration of magnetism with variablerange interactions in a quantum simulator, Science $\mathbf{3 4 0}$, 583 (2013).

[26] C. Senko, J. Smith, P. Richerme, A. Lee, W. Campbell, and C. Monroe, Coherent imaging spectroscopy of a quantum many-body spin system, Science 345, 430 (2014).

[27] S. Bose, Quantum Communication through an Unmodulated Spin Chain, Phys. Rev. Lett. 91, 207901 (2003).

[28] P. Cappellaro, C. Ramanathan, and D. G. Cory, Simulations of Information Transport in Spin Chains, Phys. Rev. Lett. 99, 250506 (2007).

[29] X. Peng, J. Du, and D. Suter, Quantum phase transition of ground-state entanglement in a Heisenberg spin chain simulated in an NMR quantum computer, Phys. Rev. A 71, 012307 (2005).

[30] M. P. Ledbetter, T. Theis, J. W. Blanchard, H. Ring, P. Ganssle, S. Appelt, B. Blümich, A. Pines, and D. Budker, Near-Zero-Field Nuclear Magnetic Resonance, Phys. Rev. Lett. 107, 107601 (2011).

[31] P. O. Boykin, T. Mor, V. Roychowdhury, F. Vatan, and R. Vrijen, Algorithmic cooling and scalable NMR quantum computers, Proc. Natl. Acad. Sci. U.S.A. 99, 3388 (2002).

[32] R. W. Adams, J. A. Aguilar, K. D. Atkinson, M. J. Cowley, P. I. Elliott, S. B. Duckett, G. G. Green, I. G. Khazal, J. López-Serrano, and D. C. Williamson, Reversible Interactions with para-Hydrogen Enhance NMR Sensitivity by Polarization Transfer, Science 323, 1708 (2009). 
[33] T. Theis, G. X. Ortiz, A. W. Logan, K. E. Claytor, Y. Feng, W. P. Huhn, V. Blum, S. J. Malcolmson, E. Y. Chekmenev, Q. Wang et al., Direct and cost-efficient hyperpolarization of long-lived nuclear spin states on universal ${ }^{15} \mathrm{~N}_{2}$-diazirine molecular tags, Sci. Adv. 2, e1501438 (2016).

[34] J. W. Blanchard, M. P. Ledbetter, T. Theis, M. C. Butler, D. Budker, and A. Pines, High-resolution zero-field NMR J-spectroscopy of aromatic compounds, J. Am. Chem. Soc. 135, 3607 (2013).

[35] D. P. Weitekamp, A. Bielecki, D. Zax, K. Zilm, and A. Pines, Zero-Field Nuclear Magnetic Resonance, Phys. Rev. Lett. 50, 1807 (1983).

[36] A. Ajoy et al. (to be published).

[37] D. Scarabelli, M. Trusheim, O. Gaathon, D. Englund, and S. J. Wind, Nanoscale engineering of closely-spaced electronic spins in diamond, Nano Lett. 16, 4982 (2016).

[38] P. Cappellaro, L. Viola, and C. Ramanathan, Coherent-state transfer via highly mixed quantum spin chains, Phys. Rev. A 83, 032304 (2011).

[39] N. Yao, L. Jiang, A. Gorshkov, P. Maurer, G. Giedke, J. Cirac, and M. Lukin, Scalable architecture for a room temperature solid-state quantum information processor, Nat. Commun. 3, 800 (2012).

[40] G. Goldstein, P. Cappellaro, J. R. Maze, J. S. Hodges, L. Jiang, A.S. Sørensen, and M. D. Lukin, EnvironmentAssisted Precision Measurement, Phys. Rev. Lett. 106, 140502 (2011).

[41] M. Schaffry, E. M. Gauger, J. J. L. Morton, and S. C. Benjamin, Proposed Spin Amplification for Magnetic Sensors Employing Crystal Defects, Phys. Rev. Lett. 107, 207210 (2011).

[42] I. Lovchinsky, A. Sushkov, E. Urbach, N. de Leon, S. Choi, K. De Greve, R. Evans, R. Gertner, E. Bersin, C. Müller et al., Nuclear magnetic resonance detection and spectroscopy of single proteins using quantum logic, Science 351, 836 (2016).

[43] A. O. Sushkov, I. Lovchinsky, N. Chisholm, R. L. Walsworth, H. Park, and M. D. Lukin, Magnetic Resonance Detection of Individual Proton Spins Using Quantum Reporters, Phys. Rev. Lett. 113, 197601 (2014).

[44] J. Kempe, A. Kitaev, and O. Regev, The complexity of the local hamiltonian problem, SIAM J. Comput. 35, 1070 (2006). 